\title{
a5-integrin is crucial for L1CAM-mediated chemoresistance in pancreatic adenocarcinoma
}

\author{
SUSANNE SEBENS MÜERKÖSTER ${ }^{1}$, JÖRN KÖTTERITZSCH ${ }^{1}$, CLAUDIA GEISMANN $^{1}$, DANIELA GAST ${ }^{2}$, \\ MARIE-LUISE KRUSE ${ }^{1}$, PETER ALTEVOGT ${ }^{2}$, ULRICH R. FÖLSCH ${ }^{1}$ and HEINER SCHÄFER ${ }^{1}$ \\ ${ }^{1}$ Clinic for General Internal Medicine, Laboratory of Molecular Gastroenterology and Hepatology, UKSH-Campus Kiel, \\ University of Kiel; ${ }^{2}$ Tumor Immunology Programme, D010, German Cancer Research Center, Heidelberg, Germany
}

Received June 27, 2008; Accepted September 3, 2008

DOI: 10.3892/ijo_00000146

\begin{abstract}
We recently showed that the adhesion molecule L1CAM (CD171) is overexpressed in pancreatic adenocarcinoma (PDAC) essentially contributing to chemoresistance of PDAC cells. In search of the mechanisms of this effect we now identified $\alpha 5$-integrin as the L1CAM ligand being essential for L1CAM-mediated chemoresistance of these highly malignant tumor cells. Thus, blockade or knock-down of $\alpha 5$-integrin in the L1CAM expressing PDAC cell lines PT45-P1res, Colo357 and Panc1 increased anti-cancer drug sensitivity. In line with the previously reported NO-dependent caspase inhibition resulting from L1CAM induced iNOS expression, the loss of chemoresistance upon $\alpha 5$-integrin inhibition was preceded by decreased iNOS expression and enhanced caspase-3/-7 activation. Accordingly, the loss of anti-cancer drug protection by $\alpha 5$-integrin inhibition could be overcome by administration of the NO-donor SNAP. Moreover, the gain of chemoresistance of parental PT45-P1 cells when transfected with L1CAM was abrogated by $\alpha 5$ integrin inhibition, whereas transfection of PT45-P1 cells with an integrin binding-deficient L1CAM mutant (L1mutRGE) did neither induce chemoresistance or iNOS expression nor conferred sensitivity to $\alpha 5$-integrin inhibition as seen upon transfection with wild-type L1CAM. Thus, mutational loss of the integrin binding site in the L1CAM molecule or the
\end{abstract}

Correspondence to: Dr Susanne Sebens Müerköster, Clinic for General Internal Medicine, Laboratory of Molecular Gastroenterology and Hepatology, UKSH-Campus Kiel, Schittenhelmstrasse 12, D-24105 Kiel, Germany

E-mail: mueerkoe@1med.uni-kiel.de

Abbreviations: iNOS, inducible nitric oxide synthetase; NO, nitric oxide; PDAC, pancreatic ductal adenocarcinoma; RT, reverse transcription; siRNA, small interfering RNA; SNAP, S-Nitroso-Nacetyl-D,L-penicillamine

Key words: L1CAM, chemoresistance, integrin, pancreatic adenocarcinoma, caspases blockade of $\alpha 5$-integrin abolished the induction of iNOS expression and chemoresistance by L1CAM, indicating that both a functional L1CAM and $\alpha 5$-integrin are indispensable of L1CAM-induced drug resistance in PDAC cells.

\section{Introduction}

In recent years, the adhesion molecule L1CAM (CD171) has attracted much attention because it is overexpressed in various tumors, e.g., in ovarian carcinoma, melanoma and colon carcinoma, being associated with short survival and poor prognosis (1-5). Moreover, L1CAM has also been detected in pancreatic adenocarcinoma (PDAC) (6).

L1CAM is a $200-220-\mathrm{kDa}$ transmembrane glycoprotein of the immunoglobulin (Ig) superfamily composed of six Ig-like domains and five fibronectin type III repeats followed by a transmembrane region and a highly conserved cytoplasmic tail (7). Previous studies demonstrated that L1CAM can interact either with itself (homophilic) or with a variety of heterophilic ligands such as integrins $\alpha 5 \beta 1, \alpha v ß 3$ or $\alpha v \beta 3$, neurocan or neuropilin-1 (8-13). In many incidences, the binding sites in the L1CAM molecule have been mapped, e.g., the RGD site(s) in the 6th Ig domain (1 in the human, 2 in the mouse) supporting $\alpha 5 \beta 1, \alpha v \beta 3$ or $\alpha v \beta 3$ integrinmediated cell binding (10-12). The 1st Ig domain can bind to the proteoglycan neurocan (14) and the VEGF-R2-coreceptor neuropilin-1 $(8,13)$.

The mechanisms by which L1CAM contributes to tumor progression are now beginning to emerge. Several studies have demonstrated that L1CAM augments tumor growth in NOD/SCID mice, enhances cell motility on extracellular matrix proteins and increases Matrigel invasion (2,15-18), thus indicating a role in tumor cell migration and metastasis. In ovarian and pancreatic carcinoma cells, L1CAM was found to mediate also resistance to apoptotic stimuli $(6,19)$, thereby accounting for the profound chemoresistance of these highly malignant tumors. Using the well characterized model system of long-term drug treated, chemoresistant PT45-P1res cells and its parental chemosensitive counterpart PT45-P1 (20), we recently demonstrated that the chemoresistant phenotype of PT45-P1res cells highly depends on L1CAM expression, itself induced by IL1ß. Moreover, we identified a mechanism by which L1CAM mediates chemoresistance in 
PDAC cells: IL1ß mediated L1CAM expression leads to the induction of the inducible nitric oxide synthetase (iNOS), resulting in enhanced levels of nitric oxide (NO) and thereby in the inactivation of caspases $(6,20)$. Meanwhile, antibodies to L1CAM or knock-down of L1CAM were shown to abolish chemoresistance $(6,19)$ and to reduce cell proliferation in vitro as well as in vivo using a xenograft mouse model (21-23). However, so far it is not known whether these L1CAMmediated processes require ligand binding.

Addressing this issue, the present study investigated the involvement of potential ligands in L1CAM-mediated chemoresistance of PDAC cells, identifying $\alpha 5$-integrin to be essential in this process.

\section{Materials and methods}

Cell lines and culture. The human PDAC cell lines PT45-P1, Colo357 and Panc1 have been described previously (24). PT45-P1 and PT45-P1res cells were kept in culture $\left(37^{\circ} \mathrm{C}\right.$, $5 \% \mathrm{CO}_{2}, 85 \%$ humidity) using RPMI-1640 medium (PAA Laboratories, Cölbe, Germany) supplemented with $1 \%$ glutamine (Invitrogen, Karlsruhe, Germany) and 10\% fetal calf serum (FCS) (Biochrom KG, Berlin, Germany). The generation of chemoresistant PT45-P1res cells deriving from PT45-P1 cells has been described elsewhere (20). Colo357 and Panc1 cells were cultured in RPMI-1640 medium (PAA Laboratories) supplemented with $1 \%$ glutamine (Invitrogen), $10 \%$ FCS (Biochrom KG) and 1\% sodium pyruvate (PAA).

Reagents. Etoposide was purchased from Bristol Myers Squibb (München, Germany). For functional blocking of integrins, a mouse IgG1 anti-human $\alpha 5$-integrin antibody, a mouse IgG1 anti-human B1-integrin antibody or an isotype matched control antibody (all from Chemicon via Millipore, Schwalbach/Ts., Germany) were used, each at a concentration of $10 \mu \mathrm{g} / \mathrm{ml}$.

Site directed mutagenesis of L1CAM. Mutagenesis of L1CAM in position D556E (resulting in RGD $\rightarrow$ RGE) was performed with the QuikChange ${ }^{\mathrm{TM}}$ Site-Directed Mutagenesis Kit according to the instructions of the manufacturer (Stratagene, Heidelberg, Germany) and as described recently (25). All constructs were verified by sequencing.

Surface staining of L1CAM, $\alpha 5$ - and $\beta 1$-integrin and flow cytometry. After washing with PBS, cells grown in 6-well culture plates were detached with $5 \mathrm{mmol} / \mathrm{l}$ EDTA/PBS and then washed with PBS. Blocking was conducted in $1 \%$ BSA/PBS for $60 \mathrm{~min}$ at room temperature. Incubation with either a monoclonal mouse IgG1 anti-L1CAM antibody [L1-11A, clone UJ127 (16)], a mouse IgG1 anti- $\alpha 5$-integrin (clone P1D6 from Santa Cruz Biotechnology, Heidelberg, Germany), a mouse IgG1 anti-ß1-integrin antibody (clone 6S6, Chemicon) or an isotype matched control antibody (R\&D Systems, Wiesbaden, Germany), each diluted 1:1000 in 1\% BSA/PBS, was performed at $4^{\circ} \mathrm{C}$ overnight. Then, cells were washed three times with PBS followed by incubation $(60 \mathrm{~min}$, $37^{\circ} \mathrm{C}$ ) with an Alexa flour 488 conjugated goat anti-mouse antibody (Dianova, Hamburg, Germany), diluted 1:500 in 1\% BSA/PBS. After washing with PBS, cells were resuspended in $500 \mu 1$ PBS and analysed by fluorescence flow cytometry.
Measurement of caspase-3/-7 activity. Cells ( $1 \times 10^{5}$ cells/ well) were grown in a 12 -well culture plate for $24 \mathrm{~h}$. Then, medium was replaced and cells were left untreated or treated as indicated. Apoptosis was determined by detection of the caspase-3/7 activity, using a homogeneous luminiscent assay following the manufacturer's instructions (Promega, Mannheim, Germany). All samples were measured in duplicates.

Cell transfection. For plasmid transfection, PT45-P1 cells were seeded into 6 -well plates $\left(2 \times 10^{5}\right.$ cells/well $)$ and grown overnight, followed by transfection with $5 \mu 1$ well DIMRIE reagent (Invitrogen) and $0.4 \mu \mathrm{g} /$ well of the following plasmids: pcDNA3.1 (mock), pcDNA3.1-L1CAM wild-type (L1wt) or pcDNA3.1-L1CAMmutRGE (L1mutRGE). Upon transfection for $18 \mathrm{~h}, 1 \mathrm{ml}$ medium containing $20 \%$ FCS was added and cells were left untreated or were treated as indicated for further $24 \mathrm{~h}$. For siRNA treatment, cells were seeded into 12 -well plates $\left(1 \times 10^{5}\right.$ cells/well) and grown overnight followed by transfection with $12 \mu \mathrm{l} /$ well RNAiFect reagent (Qiagen, Hilden, Germany) and $0.4 \mu \mathrm{l} /$ well of either Stealth negative control siRNA, Stealth $\alpha 5$-integrin siRNA or Stealth neuropilin-1 siRNA (each $20 \mu \mathrm{M}$, Invitrogen). After overnight transfection, cells were either left untreated or treated as indicated for further $24 \mathrm{~h}$.

Western blotting. Preparation of whole cell lysates as well as gel electrophoresis and immunoblotting have been described previously $(6,26)$. For detection of L1CAM, a monoclonal antibody (clone UJ127 from Acris Antibodies) was diluted $1: 1000$ in $5 \%$ non-fat milk powder and $0.05 \%$ Tween in TBS (blotto-TBST) and incubated overnight at $4^{\circ} \mathrm{C}$. For the detection of $\alpha 5$ - and $\beta 1$-integrin, polyclonal goat antibodies (both from Santa Cruz Biotechnology) were each diluted 1:200 in blotto-TBST. Soluble neuropilin-1 was detected by a polyclonal rabbit antibody (Zymed via Invitrogen) diluted 1:250 in blotto-TBST. For detection of caspases, the following antibodies (all from Cell Signaling via New England Biolabs, Frankfurt a.M., Germany) were used at the indicated dilutions: anti-caspase-3 (proenzyme), 1:1000 in blotto-TBST; anticleaved caspase-3, 1:250 in blotto-TBST; anti-caspase-7 (pro- and cleaved enzyme), 1:1000 in blotto-TBST; anticaspase-8 (pro- and cleaved enzyme), 1:1000 in 5\% BSA (Serva) in TBST; anti-caspase-9 (proenzyme), 1:1000 in blotto-TBST; anti-caspase-9 (cleaved), 1:250 in blottoTBST. iNOS was detected by a polyclonal rabbit antihuman iNOS antibody (BD Biosciences, Heidelberg, Germany), diluted 1:1000 in blotto-TBST. As control of equal protein load, a polyclonal rabbit antibody for HSP90 (Santa Cruz Biotechnology) was diluted 1:2000 in blottoTBST. For detection of the primary antibodies, anti-rabbit, anti-goat and anti-mouse HRP-linked antibodies (Cell Signaling), respectively, were used at a dilution of 1:2000 in blotto-TBST.

Real-time PCR. Total RNA ( $2 \mu \mathrm{g})$ was reverse-transcribed into single-stranded cDNA, as described previously (27). $1.3 \mu 1$ of cDNA and $0.2 \mu \mathrm{mol} / 1$ gene-specific primers were adjusted with RNAse-free water to a volume of $10 \mu 1$. To this mixture, $10 \mu 1$ of iQ SYBR Green Supermix (Bio-Rad) was 
added. The primer sequences and the PCR conditions for the detection of iNOS were: forward, CATAGAGATGGCCTGT CCTTGGA; reverse, TCCAGCTTGACCAGAGATTCTGG; $95^{\circ} \mathrm{C} / 3 \mathrm{in} ; 95^{\circ} \mathrm{C} / 1 \mathrm{~min}, 62^{\circ} \mathrm{C} / 1 \mathrm{~min}, 72^{\circ} \mathrm{C} / 20 \mathrm{sec}$ for 3 cycles; $95^{\circ} \mathrm{C} / 1 \mathrm{~min}, 61^{\circ} \mathrm{C} / 1 \mathrm{~min}, 72^{\circ} \mathrm{C} / 20 \mathrm{sec}$ for 3 cycles; $95^{\circ} \mathrm{C} / 1 \mathrm{~min}$, $60^{\circ} \mathrm{C} / 1 \mathrm{~min}, 72^{\circ} \mathrm{C} / 20 \mathrm{sec}$ for 3 cycles; $95^{\circ} \mathrm{C} / 1 \mathrm{~min}, 58^{\circ} \mathrm{C} / 1 \mathrm{~min}$, $72^{\circ} \mathrm{C} / 20 \mathrm{sec}$ for 36 cycles and $72^{\circ} \mathrm{C} / 10 \mathrm{~min}$. For normalization of the mRNA content, $B$-actin was amplified in parallel using primers from Takara (via Lonza, Potsdam, Germany). The real-time PCR was performed using a MyiQ Single Color Real-time PCR Detection System (Bio-Rad). Data were collected during annealing steps and were further analysed by using the i-Cycler iQ Optical system software (Bio-Rad). All samples were analysed in duplicates.

Statistical analysis. Data are presented as mean \pm standard deviations and were analyzed by Student's t-test using the simple interactive statistical analysis (SISA) program (http://home.clara.net/sisa/). A p $<0.05$ was considered as statistically significant.

\section{Results}

Chemosensitive PT45-P1 and chemoresistant PT45-P1res cells differentially express L1CAM, but exhibit a similar expression of potential LICAM ligands. Recently, we have shown that the PDAC cell line PT45-P1 becomes chemoresistant (PT45-P1res) upon long-term drug treatment (20). Moreover, PT45-P1res cells are characterized by an elevated expression of L1CAM accounting for acquired drug resistance as similarly shown by diminished Annexin $\mathrm{V}$ binding and caspase-3/7 activity (6). To investigate whether L1CAMmediated chemoresistance depends on heterophilic ligands, PT45-P1 and PT45-P1res cells were screened by Western blotting for the expression of neuropilin- 1 as well as of $\alpha 5-$, $\alpha v_{-}, \beta 1-, \beta 3-$ and B5-integrins, all being potential ligands of L1CAM. Whereas expression of $\alpha \mathrm{V}-, \beta 3$ - and 35 -integrin could not be found in either cell line (data not shown), PT45P1res and PT45-P1 cells showed similar expression levels of $\alpha 5-, \beta 1$-integrin and of neuropilin-1 (Fig. 1A). Interestingly, in both cell lines only expression of the soluble form of neuropilin-1 (90 kDa) but not of the $130-\mathrm{kDa}$ membranebound form (data not shown) could be detected. According to our previous findings, chemoresistant PT45-P1res cells exhibited an elevated expression of L1CAM compared to its chemosensitive counterpart PT45-P1 (Fig. 1A). Fluorescence flow cytometry confirmed cell surface expression of L1CAM only in PT45-P1res cells and $\alpha 5$ - as well as $\beta 1$-integrin expression in both cell lines (Fig. 1B).

Blocking or knock-down of a5-integrin increases chemosensitivity in PT45-P1res cells. Since neuropilin-1 has been described to promote chemoresistance in certain PDAC cell lines (28), we analysed whether this potential L1CAM ligand is involved in L1CAM-mediated chemoresistance of PT45P1res cells. Transfection of these cells with neuropilin-1 specific siRNA clearly reduced neuropilin-1 expression and did not affect that of L1CAM (Fig. 2A), confirming specificity of the neuropilin-1 siRNA. Under these conditions, the apoptotic response towards etoposide treatment $(20 \mu \mathrm{g} / \mathrm{ml}$ for
A)

L1CAM $(220 / 200 \mathrm{kD})>$

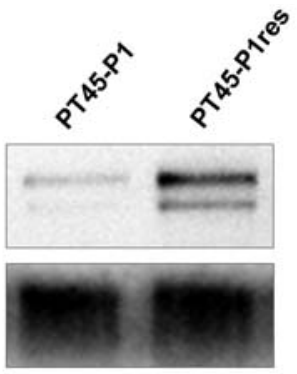

$\beta 1$-integrin $(138 \mathrm{kD})>$

Neuropilin-1 $(90 \mathrm{kD})>$

HSP90 $(90 \mathrm{kD})>$
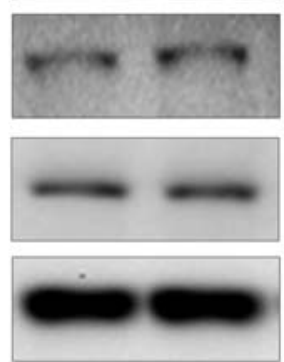

B)

PT45-P1

PT45-P1res
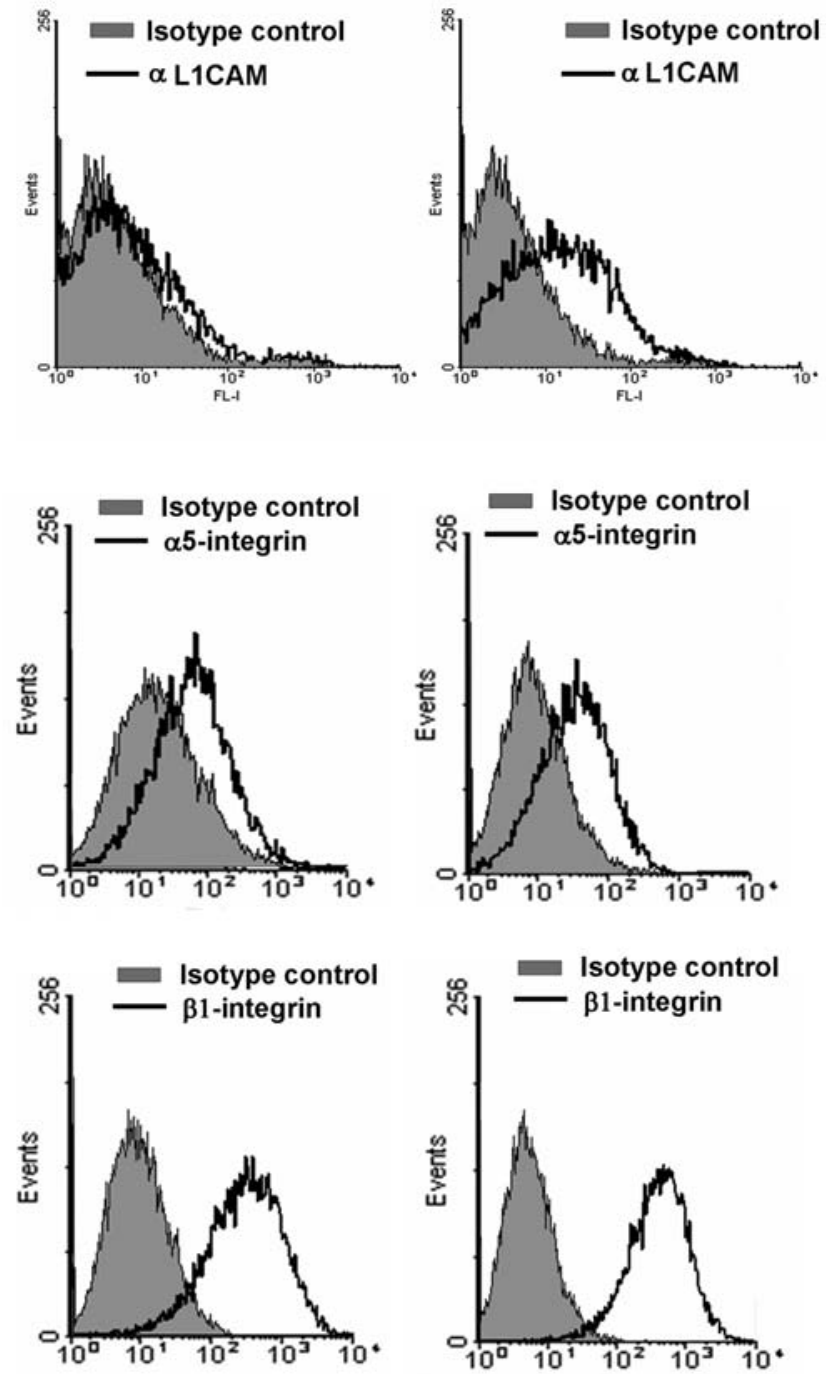

Figure 1. Expression of L1CAM and L1CAM ligands in PT45-P1 and PT45-P1res cells. (A) Cellular lysates were subjected to Western blotting detecting L1CAM, soluble neuropilin-1, $\alpha 5$ - and $\beta 1$-integrin. HSP90 was detected as control for equal protein load. (B) Representative histograms from fluorescence flow cytometry of surface stainings of L1CAM, $\alpha 5$ - and B1-integrin, each compared to staining with an isotype matched control antibody. 

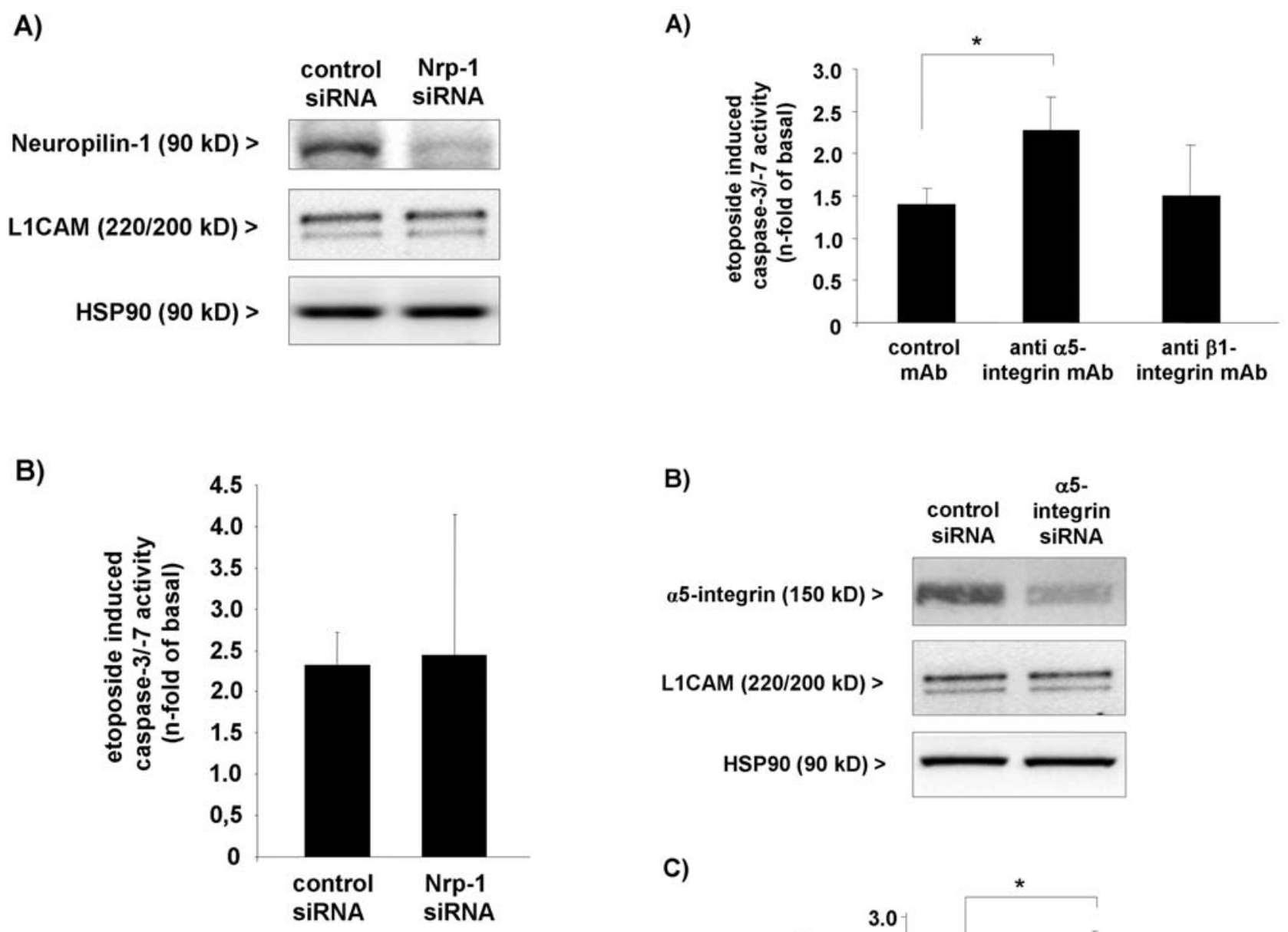

Figure 2. Neuropilin-1 is not involved in chemoresistance in PT45-P1res cells. (A) PT45-P1res cells were transfected with control siRNA or with a neuropilin-1 specific siRNA. After $48 \mathrm{~h}$, Western blotting for the detection of neuropilin-1, L1CAM and of HSP90, used as a control for equal protein load, was performed. (B) In parallel, siRNA transfected PT45-P1res cells were treated with $20 \mu \mathrm{g} / \mathrm{ml}$ etoposide or not for $24 \mathrm{~h}$ and caspase-3/-7 activity was determined. Data are expressed as etoposide induced caspase-3/-7 activity (n-fold of basal). Means \pm SD from 6 independent experiments are shown.

$24 \mathrm{~h}$ ) remained unchanged as indicated by a similar activation of caspase-3/-7 compared to control transfected cells (Fig. 2B), thus excluding neuropilin-1 as potential ligand of L1CAMmediated chemoresistance.

To analyse whether $\alpha 5$ - and 81 -integrin are involved in the mediation of chemoresistance, drug-induced apoptosis was measured in PT45-P1res cells subject to treatment with blocking antibodies against the respective integrin. Although B1-integrin blocking antibodies abolished migration of PT45-P1res cells (data not shown), no chemosensitizing effect was observed in these cells (Fig. 3A). In contrast, blocking of $\alpha 5$-integrin significantly increased chemosensitivity, as indicated by the elevated caspase-3/-7 activity in response to etoposide which rose by $>50 \%$ compared to PT45-P1res cells treated with an isotype matched control antibody (Fig. 3A). To confirm the involvement of $\alpha 5$-integrin in the chemoresistant phenotype of PT45-P1res cells, expression of $\alpha 5$-integrin was reduced by transfection of $\alpha 5$-integrin siRNA, not affecting expression of L1CAM (Fig. 3B). Under these conditions, etoposide induced

Figure 3. Blocking or knock-down of $\alpha 5$-integrin increases chemosensitivity in PDAC cell lines. (A) PT45-P1res cells were either left untreated or were treated with $20 \mu \mathrm{g} / \mathrm{ml}$ etoposide in the presence of either an anti- $\alpha 5$-integrin antibody, an anti-31-integrin antibody or an isotype matched control antibody (each at $10 \mu \mathrm{g} / \mathrm{ml})$. After $24 \mathrm{~h}$, caspase-3/-7 activity was determined $(\mathrm{n}=3)$. (B) PT45-P1res cells were transfected with control siRNA or with $\alpha 5$-integrin specific siRNA. After $48 \mathrm{~h}$, Western blotting for the detection of $\alpha 5$-integrin, L1CAM and of HSP90, used as a control for equal protein load, was performed. (C) In parallel, siRNA transfected PT45-P1res cells were treated with $20 \mu \mathrm{g} / \mathrm{ml}$ etoposide or not for $24 \mathrm{~h}$ and caspase-3/-7 activity was determined $(n=4)$.

caspase-3/-7 activity was increased in PT45-P1res cells (Fig. 3C) to a similar extent as seen upon treatment with blocking antibodies (Fig. 3A), indicating that the chemoresistant phenotype of these cells is dependent on a5-integrin. Furthermore, knock-down (Fig. 3D) and blocking of $\alpha 5$ integrin both similarly increased drug induced caspase-3/-7 activity in the two L1CAM expressing PDAC cell lines Colo357 and Panc1 (Fig. 3E), supporting a general role of $\alpha 5$-integrin in chemoresistance of PDAC cells. 
D)

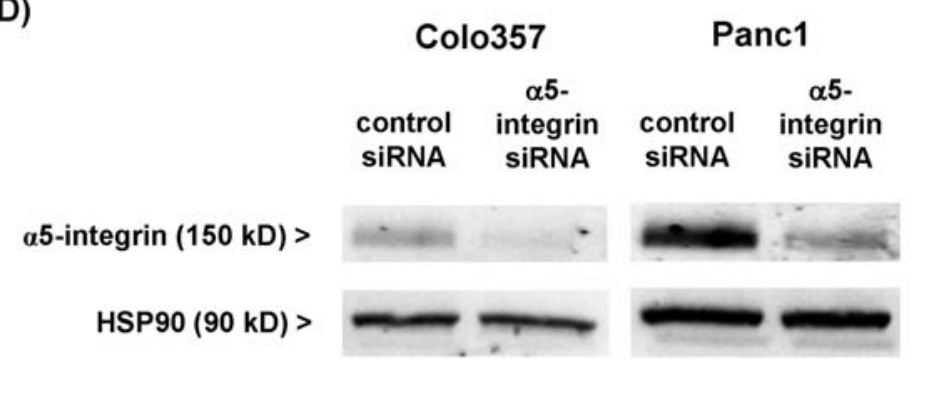

E)
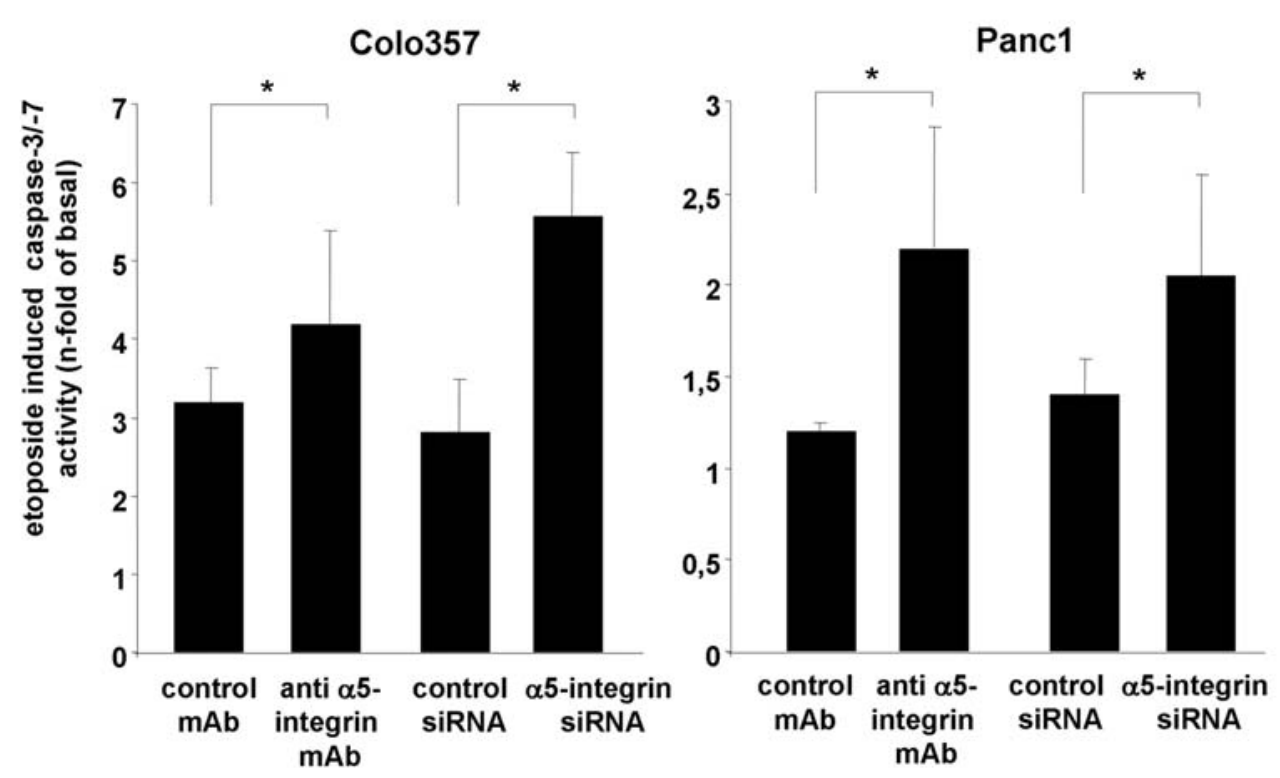

Figure 3 (continued). (D) Colo357 and Panc1 cells were transfected with control siRNA or with $\alpha 5$-integrin specific siRNA. After 48 h, Western blotting for the detection of $\alpha 5$-integrin and of HSP90, the latter used as a control for equal protein load, was performed. (E) Colo357 and Panc1 cells were either transfected as described in D or were left untransfected, the latter either treated with an isotype matched control antibody or with an anti- $\alpha 5$-integrin antibody (each at $10 \mu \mathrm{g} / \mathrm{ml}$ ). Transfected and antibody treated cells were either left untreated or stimulated with $20 \mu \mathrm{g} / \mathrm{ml}$ etoposide. After $24 \mathrm{~h}$, caspase-3/-7 activity was determined $(n=3)$. Data are expressed as etoposide induced caspase-3/-7 activity ( $\mathrm{n}$-fold of basal). Means \pm SD from 3 (A and E) and 4 (C) independent experiments are shown. ${ }^{\mathrm{p}}<0.05$.

a5-integrin is involved in the induction of iNOS in L1CAM expressing PT45-Plres cells. We previously showed that acquired chemoresistance results from an increased expression of the inducible nitric oxide synthetase (iNOS) and an elevated secretion of NO leading to the inhibition of caspases (20). Since L1CAM is essential for iNOS induction, thereby accounting for caspase inhibition, we looked whether $\alpha 5$-integrin is involved in this mechanism of L1CAM mediated chemoresistance. Blocking of $\alpha 5$-integrin (data not shown) or knock-down of $\alpha 5$-integrin expression by siRNA transfection clearly diminished the expression of iNOS in PT45-P1res cells as demonstrated by real-time PCR analysis (Fig. 4A) and by Western blot analysis (Fig. 4B). In accordance with these findings, the enhanced drug induced caspase-3/-7 activity that was seen upon knock-down of $\alpha 5$-integrin could be reversed in these cells by addition of the NO donor SNAP. As shown in Fig. 4C, administration of SNAP almost completely diminished the etoposide-induced caspase-3/-7 activity in $\alpha 5$-integrin siRNA treated PT45-P1res cells. a5-integrin is involved in the inhibition of caspases in L1CAM expressing PT45-P1res cells. Given a role of $\alpha 5$-integrin in the induction of iNOS expression, as a consequence one would expect that this L1CAM ligand is also involved in the inhibition of caspases. Thus, we next analysed the effect of $\alpha 5$-integrin knock-down on the activation of caspases in chemoresistant PT45-P1res cells. Whereas knock-down of $\alpha 5$-integrin did not affect the expression level of the procaspases-3, -7, -8 and -9 upon etoposide treatment (Fig. 5), the cleavage of these caspases was clearly enhanced, thus indicating their increased activation under these conditions (Fig. 5).

\section{L1CAM mediated chemoresistance requires an intact integrin} binding site. To further support that L1CAM-mediated chemoresistance requires $\alpha 5$-integrin, we used a L1CAM variant bearing a mutation within the RGD site in the 6th Ig domain (L1mutRGE) that affects integrin binding. L1CAM negative PT45-P1 cells were transfected with either a control 
A)

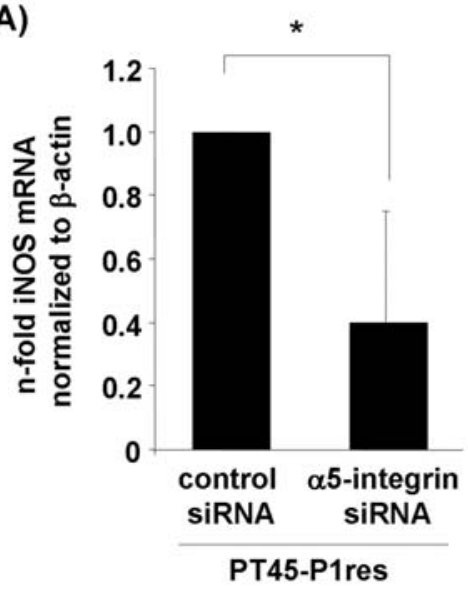

C)

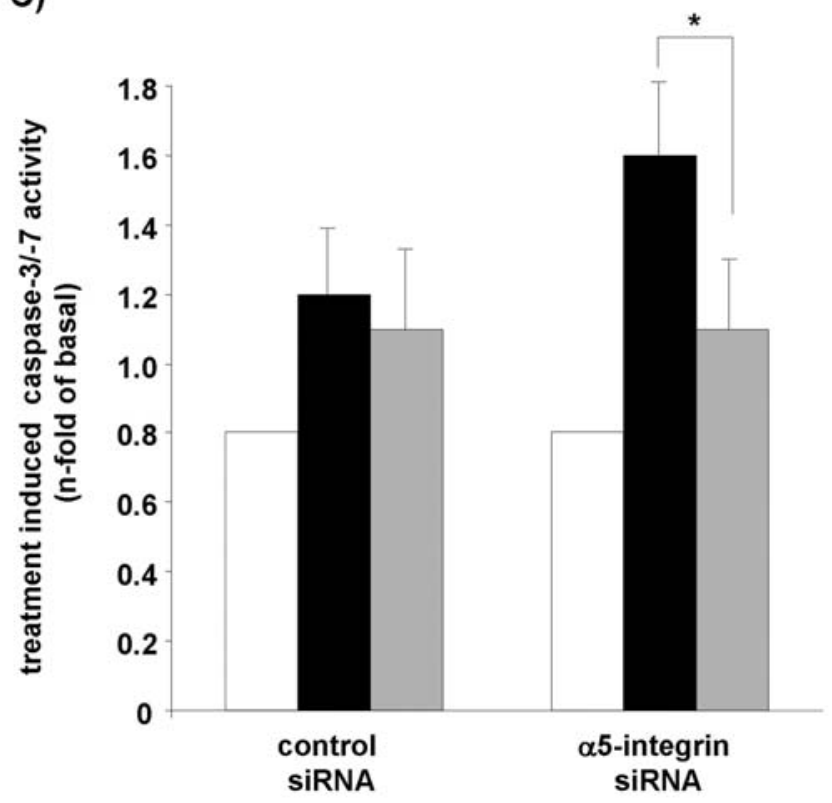

B)

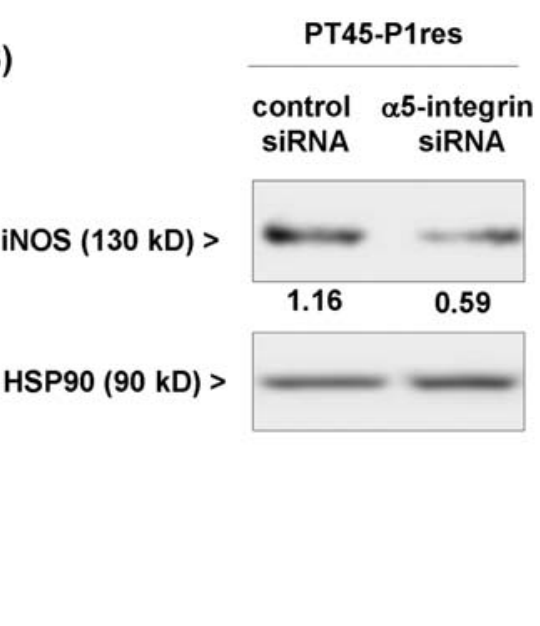

$\square$ SNAP
eto
$\square$ SNAP+ eto

Figure 4. $\alpha 5$-integrin is involved in the induction of iNOS in L1CAM expressing PT45-P1res cells. PT45-P1res cells were transfected with control or $\alpha 5$-integrin specific siRNA. (A) RNA from transfected PT45-P1res cells (24 h) was subjected to RT and subsequent real-time PCR using primers specific for iNOS and for $\beta$-actin, used for normalization of mRNA content. Normalized mRNA levels of iNOS in PT45-P1 cells, either upon $\alpha 5$-integrin blocking or upon $\alpha 5$-integrin knock-down, were related to normalized mRNA levels in respective control treated cells. (B) Cellular lysates of siRNA transfected PT45-P1res cells were subjected to Western blotting detecting iNOS and HSP90, used as control for equal protein load. Numbers displayed below the images represent the band intensity level obtained from densitometric analysis and subsequent normalization to HSP90. (C) siRNA transfected PT45-P1res cells were left untreated or were treated with $200 \mu \mathrm{mol} / \mathrm{l} \mathrm{SNAP,} 20 \mu \mathrm{g} / \mathrm{ml}$ etoposide or with a combination of both. After $24 \mathrm{~h}$, caspase-3/-7 activity was determined. Data are expressed as treatment induced caspase-3/-7 activity (n-fold of basal). Means \pm SD from three independent experiments are shown. ${ }^{*} \mathrm{p}<0.05$.

plasmid (pcDNA3.1), wild-type (L1wt) or mutant L1CAM (L1mutRGE). After transfection, comparable amounts of both L1CAM proteins could be detected in these cells by Western blot analysis (Fig. 6A) as well as by L1CAM staining and subsequent flow cytometry analysis (Fig. 6B). Transfection of chemosensitive PT45-P1 cells with the wild-type form of L1CAM significantly decreased caspase-3/-7 activation upon etoposide treatment by $50 \%$, whereas expression of mutant L1CAM hardly affected the drug induced caspase-3/-7 activation in these cells (Fig. 6C).

In line with these findings, antibody blockage of $\alpha 5$-integrin only reversed the chemoresistant phenotype of PT45-P1 cells gained after transfection with wild-type L1CAM, whereas chemosensitivity of L1mutRGE expressing cells was not altered. As shown in Fig. 6D, etoposide induced caspase-3/-7 activity was completely abolished in L1wt expressing PT45-P1 cells in the presence of $\alpha 5$-integrin blocking antibodies, supporting the hypothesis that $\alpha 5$-integrin is crucial for L1CAM-mediated chemoresistance in PDAC cells.

Expression of iNOS is elevated in PT45-P1 cells expressing the wild-type but not the RGE mutant form of LICAM. Next, PT45-P1 cells were transfected with either L1CAM construct in the absence or presence of $\alpha 5$-integrin blocking antibodies and 


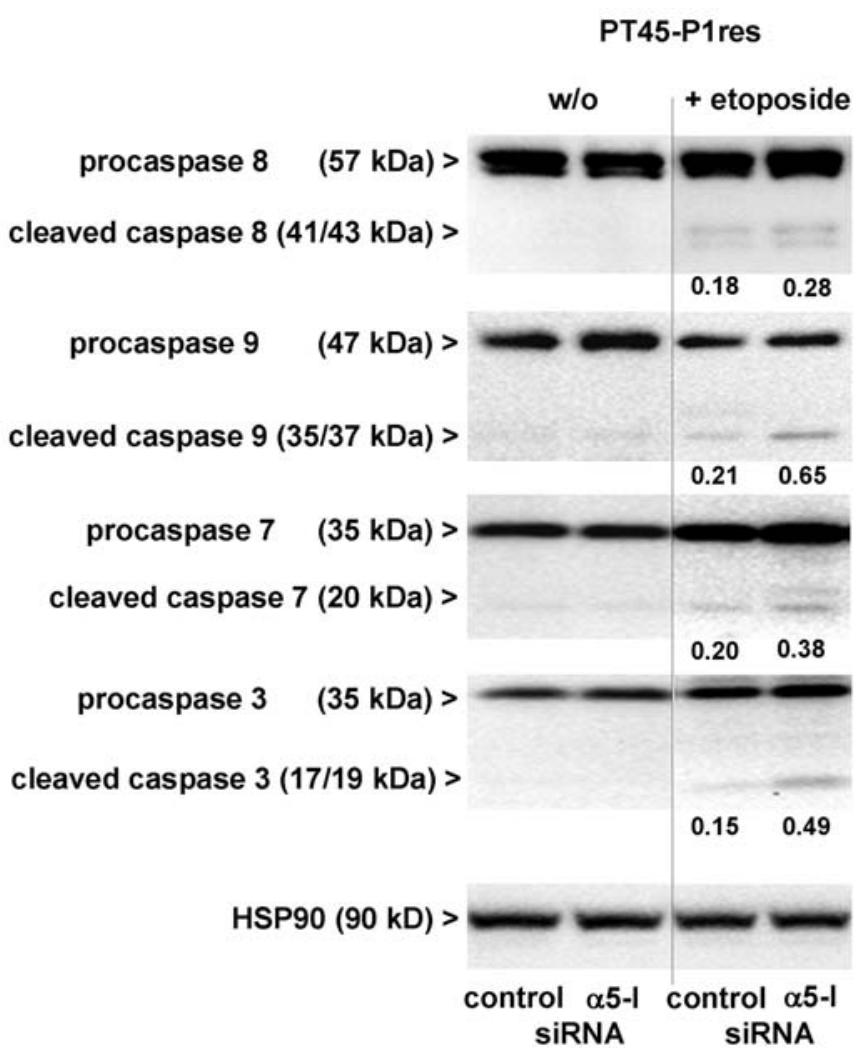

Figure 5. $\alpha 5$-integrin is involved in the inhibition of caspases in L1CAM expressing PT45-P1res cells. PT45-P1res cells were either transfected with control siRNA or with $\alpha 5$-integrin specific siRNA ( $\alpha 5$-I siRNA) followed by treatment with $20 \mu \mathrm{g} / \mathrm{ml}$ etoposide for $48 \mathrm{~h}$ or not. Cellular lysates were submitted to Western blotting using antibodies for the detection of full length (procaspase) and cleaved caspase-3, -7, -8 and -9. A HSP90 antibody was used as a control for equal protein load. Numbers displayed below the images represent the band intensity level obtained from densitometric analysis and subsequent normalization to HSP90.

were analysed for iNOS induction. In line with the findings in PT45-P1res cells, transfection of PT45-P1 cells with L1wt significantly increased expression of iNOS as demonstrated by real-time PCR (Fig. 7A) and Western blot analysis (Fig. 7C). In contrast, this effect was not observed after transfection with L1mutRGE (Fig. 7A and C) indicating that L1CAM requires a functional integrin binding site to induce iNOS expression. Furthermore, blocking of $\alpha 5$-integrin clearly abrogated the increased iNOS expression level in PT45-P1 cells transfected with L1wt (Fig. 7B and D), whereas iNOS expression in L1mutRGE expressing PT45-P1 cells remained unaffected. These data clearly point to a combined role of L1CAM and $\alpha 5$-integrin in the induction of iNOS in PT45-P1 cells.

\section{Discussion}

Enhanced L1CAM expression could be detected in PDAC accounting for acquired (6) as well as for innate (unpublished data) chemoresistance in PDAC cells. Regarding the role of L1CAM in carcinogenesis, so far only the impact of L1CAM on tumor cell migration and metastasis has been described in some detail $(2,15,16,29)$. Besides L1CAM itself, both in its membrane-bound and soluble form, other adhesion molecules known to be involved in tumor cell migration have been identified as L1CAM ligands, e.g., the integrins $\alpha 5 \beta 1$, $\alpha v ß 1$ and $\alpha v \beta 3(30,31)$. Thus, binding to and signaling through integrins might be one mechanism by which L1CAM promotes tumor cell migration (16), the upregulation of integrin expression, as demonstrated for 33 -integrin, might be another (18). However, little is known about the exact mechanisms how L1CAM mediates cell migration, in particular which ligands and cellular signals are involved. This even more applies for the mechanisms underlying L1CAM-mediated chemoresistance. Several studies have already shown that integrin dependent adhesion to extracellular matrix proteins or to adjacent stroma cells confers apoptosis protection towards cytostatic drugs, death ligands or ionizing radiation in various tumor cells (32-34). Since integrins as well as neuropilin-1 are expressed in $\operatorname{PDAC}(28,35)$ and also represent potential ligands of L1CAM, it is tempting to speculate that one of these ligands is involved in L1CAMmediated chemoresistance of PDAC cells. We have already shown that PT45-P1res cells acquiring a chemoresistant phenotype upon long-term drug treatment are characterized by an elevated expression of L1CAM compared to the chemosensitive parental cell line PT45-P1 (6). Moreover, L1CAM expression in PT45-P1res cells accounts for increased expression of iNOS and elevated NO levels resulting in caspase inhibition (20). Since it has been shown that proteolytic cleavage of L1CAM is dispensable for chemoresistance in PDAC as well as in ovarian carcinoma cells $(6,19)$, soluble L1CAM can be excluded as potential ligand in this context. Instead, homophilic interaction of membranebound L1CAM with other membrane-bound L1CAM molecules, either on the same cell or on adjacent cells, might be crucial for mediation of chemoresistance. This also applies for heterophilic L1CAM ligands, including $\alpha 5$ - and $\beta 1$-integrin as well as the soluble form of neuropilin-1 that have been detected in PT45-P1res and PT45-P1 cells. It can be assumed that L1CAM expressing tumor cells interact with these ligands on adjacent tumor or stromal cells (e.g., myofibroblasts). Contact to neighbouring cells could favour a bidirectional signaling via L1CAM and its binding to the respective ligand because ligand and receptor are often expressed by the same cell. So far, it is not known whether the anti-apoptotic signaling originates from L1CAM or from its ligand.

Wey et al demonstrated that transfection of PDAC cells with neuropilin-1 conferred drug resistance and, in turn, knock-down of neuropilin-1 increased chemosensitivity of PDAC cells (28), pointing to a role for neuropilin-1 in chemoresistance of this tumor entity. However, knock-down of neuropilin-1 in PT45-P1res cells did not affect drug induced apoptosis, indicating that neuropilin-1 is apparently not involved in the mediation of chemoresistance in this cell line. One explanation might be that soluble neuropilin-1, as detected in PT45-P1res cells, does not confer chemoresistance, at all. In contrast, blocking and knock-down of $\alpha 5$-integrin clearly increased the chemosensitivity not only of PT45P1res cells but also of the PDAC cell lines Colo357 and Panc1 pointing to an essential role of this integrin in the mediation of chemoresistance in L1CAM expressing PDAC cells. Moreover, the abrogation of $\alpha 5$-integrin expression was associated with a decreased expression of iNOS and 
A)

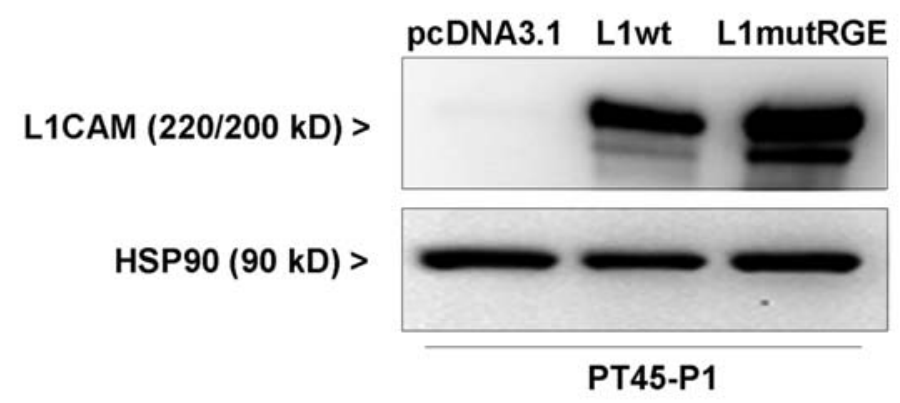

B)
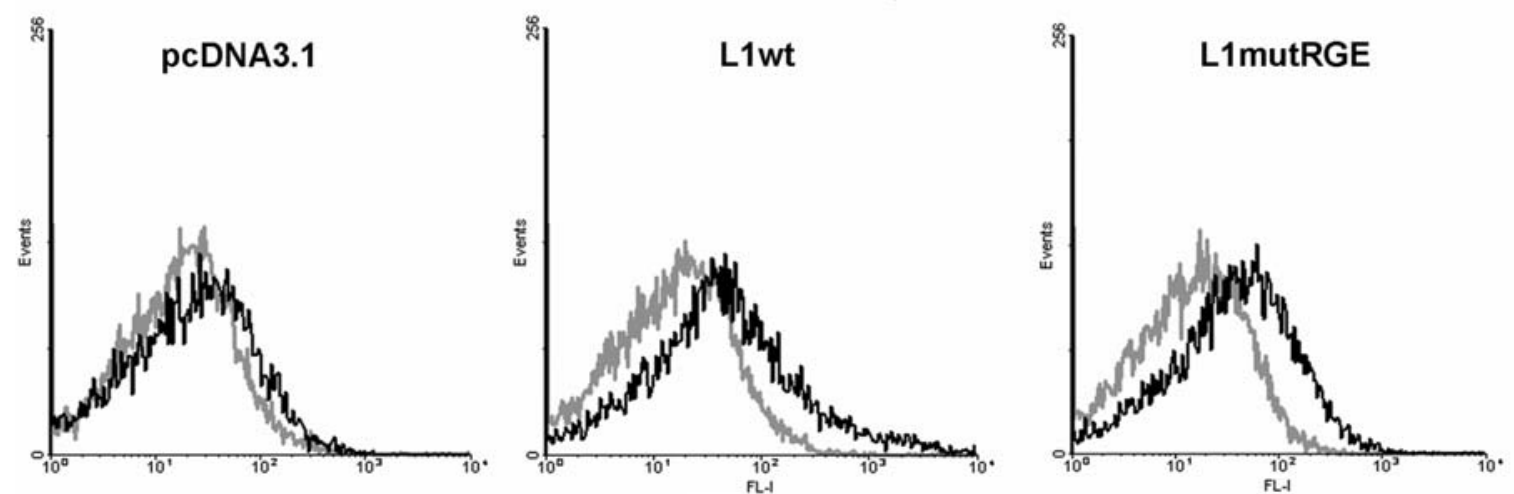

C)

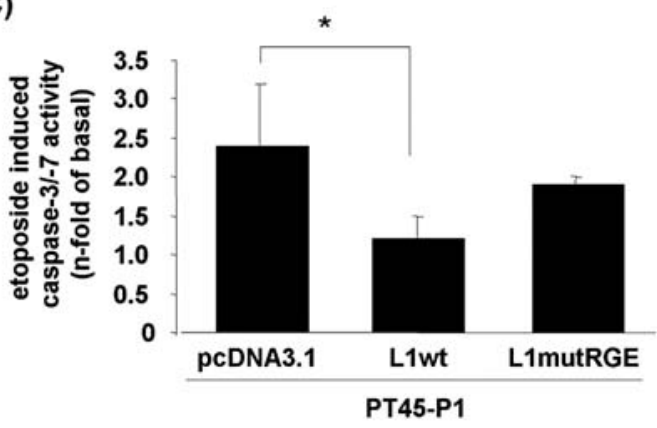

D)

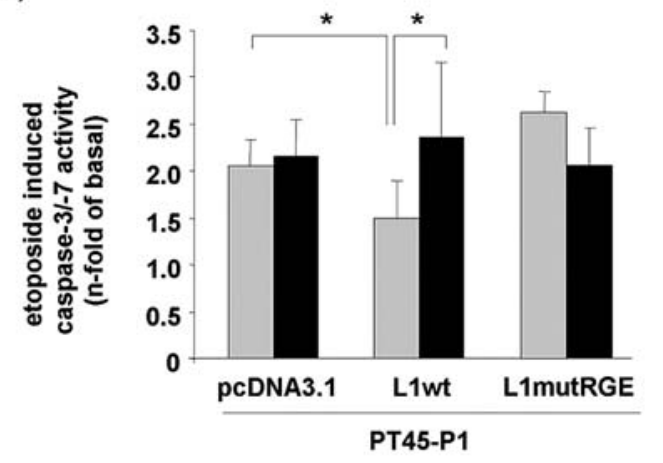

Figure 6. L1CAM mediated chemoresistance in PT45-P1 cells requires an intact integrin binding site. PT45-P1 cells were either transfected with an empty vector (pcDNA3.1), with wild-type (L1wt) or with mutant L1CAM (L1mutRGE). (A) Fourty-eight hours after transfection, cellular lysates were subjected to Western blotting for the detection of L1CAM and of HSP90, used as control of equal protein load. (B) Transfected PT45-P1 cells were subjected to L1CAM immunostaining (L1-11A antibody) or to staining with an isotype matched control antibody followed by fluorescence flow cytometry analysis. One representative histogram of each is shown. (C) After overnight transfection, cells were either left untreated or were treated with $20 \mu \mathrm{g} / \mathrm{ml}$ etoposide for $24 \mathrm{~h}$ followed by caspase-3/-7 assay. Data are expressed as etoposide induced caspase-3/-7 activity (n-fold of basal). (D) Transfected PT45-P1 cells were either left untreated or were treated with $20 \mu \mathrm{g} / \mathrm{ml}$ etoposide in the presence of either an anti- $\alpha 5$-integrin antibody or an isotype matched control antibody (each at $10 \mu \mathrm{g} / \mathrm{ml}$ ). After $24 \mathrm{~h}$, caspase-3/-7 activity was determined. Data are expressed as etoposide induced caspase-3/-7 activity (n-fold of basal). Means \pm SD from 4 (C) and 3 (D) independent experiments are shown. ${ }^{*} \mathrm{p}<0.05$.

with an increased activation of caspases, thus indicating that $\alpha 5$-integrin mediated chemoresistance involves a NOdependent inhibition of caspases. Recently, Estrugo et al (32) demonstrated that transfection of human leukemia cells with $\beta 1$-integrin confers apoptosis resistance to cytostatic drugs, activation of Fas or ionizing radiation via inhibition of control mAb anti $\alpha 5$-integrin mAb caspase- 8 . Since $\beta 1$ often dimerizes with the $\alpha 5$-subunit, one would expect that inhibition of the $\beta 1$-subunit also leads to 
A)

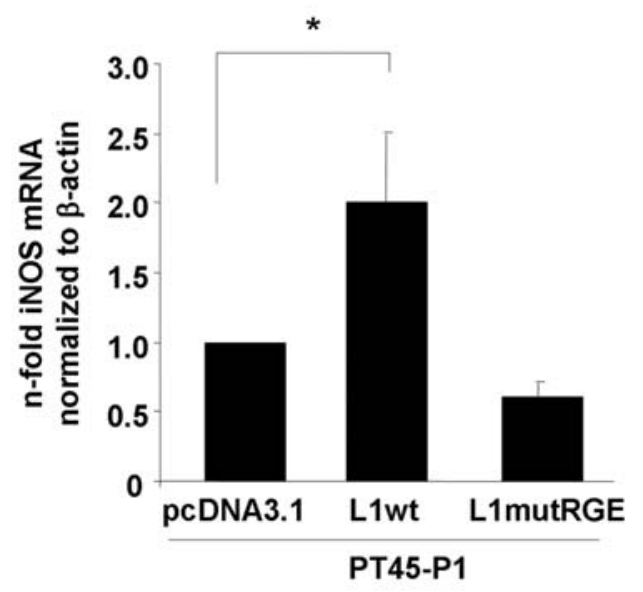

C)

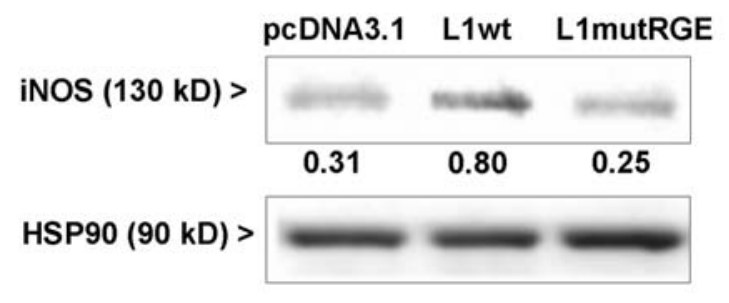

D)

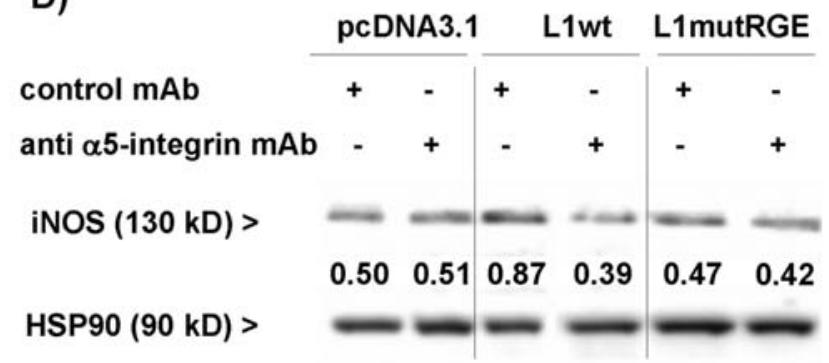

an abrogation of the chemoresistant phenotype of PT45-P1res cells. Interestingly, we observed an increasing effect by inhibition of $\alpha 5$-integrin but not of $\beta 1$-integrin on caspase activation and chemosensitivity in PT45-P1res cells. This might be explained by the fact that $\beta 1$ can dimerize with most of the other $\alpha$-subunits as well $(36,37)$, predominating over $\alpha 5 \beta 1$ integrin formation as described e.g., for $\alpha 6 \beta 1$ expression in PDAC (37-39). To support the involvement of $\alpha 5$-integrin particularly in L1CAM-mediated chemoresistance, we made use of a L1CAM variant (L1mutRGE) bearing a mutated integrin binding site in the 6th Ig domain. Transfection of chemosensitive PT45-P1 cells with wild-type L1CAM (L1wt) increased expression of iNOS along with a reduced caspase activation upon etoposide treatment. In contrast, chemosensitivity as well as iNOS expression remained unchanged in L1mutRGE expressing PT45-P1 cells. These
Figure 7. Expression of iNOS is elevated in PT45-P1 cells expressing the wild-type but not the mutant form of L1CAM. PT45-P1 cells were either transfected with an empty vector (pcDNA3.1), with wild-type L1CAM (L1wt) or with mutant L1CAM (L1mutRGE). In some experiments (B and D), cells were treated with $10 \mu \mathrm{g} / \mathrm{ml}$ of either isotype matched control antibody or $\alpha 5$ integrin antibody for $24 \mathrm{~h}$. (A) RNA from transfected PT45-P1 cells was subjected to RT and subsequent real-time PCR using primers specific for iNOS and for $\beta$-actin, used for normalization of mRNA content. Normalized mRNA levels of iNOS in L1CAM transfected PT45-P1 cells were related to normalized mRNA levels in pcDNA3.1 transfected cells. Means \pm SD from three independent experiments are shown. ${ }^{*}<0.05$. (B) RNA from transfected and antibody treated PT45-P1 cells was subjected to RT and subsequent real-time PCR using primers specific for iNOS and for $\beta$-actin, used for normalization of mRNA content. Normalized mRNA levels of iNOS in control or L1CAM transfected PT45-P1 cells upon treatment with $\alpha 5$-integrin antibody were related to the normalized mRNA levels of iNOS in the respective transfected cells upon treatment with the control antibody. Results from one representative out of three experiments are shown. (C and D) Cellular lysates from transfected cells either left untreated (C) or exposed to either control antibody or $\alpha 5$-integrin antibody (each at $10 \mu \mathrm{g} / \mathrm{ml}$ ) (D), were subjected to Western blotting detecting iNOS and HSP90 used as control for equal protein load. Numbers displayed below the images represent the band intensity level obtained from densitometric analysis and subsequent normalization to HSP90.

data already point to the necessity of a functional integrin binding site in the L1CAM molecule. Moreover, it is important to note that blocking of $\alpha 5$-integrin reduced expression of iNOS and abolished the chemoresistant phenotype only in L1wt expressing PT45-P1 cells but not in PT45-P1 cells expressing either no L1CAM at all (parental or mock) or L1CAM with a non-functional integrin binding site (L1mutRGE). Thus, L1CAM failes to induce iNOS mediated chemoresistance in PT45-P1 cells if lacking this integrin binding site as well as if $\alpha 5$-integrin is blocked or $\alpha 5$-integrin expression is silenced. In conclusion, this study deepens the understanding of L1CAM-mediated chemoresistance in PDAC and provides evidence that $\alpha 5$-integrin is of pivotal importance in this process. Whether the activated signaling cascade leading to iNOS induction and drug resistance originates from L1CAM or from $\alpha 5$-integrin is a matter of ongoing studies. 


\section{Acknowledgements}

This study was supported by the German Research Society DFG Scha 677/7-3 (H.S.) and a grant of the medical faculty of the University of Kiel (S.S.M.). The authors whish to thank Maike Grossmann and Maike Witt for their excellent technical support.

\section{References}

1. Fogel M, Gutwein P, Mechtersheimer S, Riedle S, Stoeck A, Smirnov A, Edler L, Ben-Arie A, Huszar M and Altevogt P: L1 adhesion molecule (CD 171) in development and progression of human malignant melanoma. Lancet 362: 869-875, 2003.

2. Gavert N, Conacci-Sorrell M, Gast D, Schneider A, Altevogt P, Brabletz T and Ben-Ze'ev A: L1, a novel target of beta-catenin signaling, transforms cells and is expressed at the invasive front of colon cancers. J Cell Biol 168: 633-642, 2005.

3. Kaifi JT, Reichelt U, Quaas A, Schurr PG, Wachowiak R, Yekebas EF, Strate T, Schneider C, Pantel K, Schachner M, Sauter G and Izbicki JR: L1 is associated with micrometastatic spread and poor outcome in colorectal cancer. Mod Pathol 20: 1183-1190, 2007.

4. Shtutman M, Levina E, Ohouo P, Baig M and Roninson IB: Cell adhesion molecule L1 disrupts E-cadherin-containing adherens junctions and increases scattering and motility of MCF7 breast carcinoma cells. Cancer Res 66: 11370-11380, 2006.

5. Thies A, Schachner M, Moll I, Berger J, Schulze HJ, Brunner G and Schumacher U: Overexpression of the cell adhesion molecule L1 is associated with metastasis in cutaneous malignant melanoma. Eur J Cancer 38: 1708-1716, 2002.

6. Sebens Muerkoster S, Werbing V, Sipos B, Debus MA, Witt M, Grossmann M, Leisner D, Kotteritzsch J, Kappes H, Kloppel G, Altevogt P, Folsch UR and Schafer H: Drug-induced expression of the cellular adhesion molecule L1CAM confers antiapoptotic protection and chemoresistance in pancreatic ductal adenocarcinoma cells. Oncogene 26: 2759-2768, 2007.

7. Moos M, Tacke R, Scherer H, Teplow D, Fruh K and Schachner M: Neural adhesion molecule L1 as a member of the immunoglobulin superfamily with binding domains similar to fibronectin. Nature 334: 701-703, 1998.

8. Castellani V, De Angelis E, Kenwrick S and Rougon G: Cis and trans interactions of L1 with neuropilin-1 control axonal responses to semaphorin 3A. EMBO J 21: 6348-6357, 2002.

9. Ebeling O, Duczmal A, Aigner S, Geiger C, Schollhammer S, Kemshead JT, Moller P, Schwartz-Albiez R and Altevogt P: L1 adhesion molecule on human lymphocytes and monocytes: expression and involvement in binding to alpha $\mathrm{v}$ beta 3 integrin. Eur J Immunol 26: 2508-2516, 1996.

10. Montgomery AM, Becker JC, Siu CH, Lemmon VP, Cheresh DA, Pancook JD, Zhao X and Reisfeld RA: Human neural cell adhesion molecule L1 and rat homologue NILE are ligands for integrin alpha v beta 3. J Cell Biol 132: 475-485, 1996.

11. Oleszewski M, Beer S, Katich S, Geiger C, Zeller Y, Rauch U and Altevogt P: Integrin and neurocan binding to L1 involves distinct Ig domains. J Biol Chem 274: 24602-24610, 1999.

12. Ruppert M, Aigner S, Hubbe M, Yagita H and Altevogt P: The L1 adhesion molecule is a cellular ligand for VLA-5. J Cell Biol 131: 1881-1891, 1995.

13. Stoeck A, Schlich S, Issa Y, Gschwend V, Wenger T, Herr I, Marme A, Bourbie S, Altevogt P and Gutwein P: L1 on ovarian carcinoma cells is a binding partner for Neuropilin-1 on mesothelial cells. Cancer Lett 239: 212-226, 2006.

14. Oleszewski M, Gutwein P, von der Lieth W, Rauch U and Altevogt P: Characterization of the L1-neurocan-binding site. Implications for L1-L1 homophilic binding. J Biol Chem 275: 34478-34485, 2000.

15. Gast D, Riedle S, Schabath H, Schlich S, Schneider A, Issa Y, Stoeck A, Fogel M, Joumaa S, Wenger T, Herr I, Gutwein P and Altevogt, P: L1 augments cell migration and tumor growth but not beta 3 integrin expression in ovarian carcinomas. Int $\mathbf{J}$ Cancer 115: 658-665, 2005.

16. Mechtersheimer S, Gutwein P, Agmon-Levin N, Stoeck A, Oleszewski M, Riedle S, Postina R, Fahrenholz F, Fogel M, Lemmon $\mathrm{V}$ and Altevogt P: Ectodomain shedding of L1 adhesion molecule promotes cell migration by autocrine binding to integrins. J Cell Biol 155: 661-673, 2001.

17. Meier F, Busch S, Gast D, Goppert A, Altevogt P, Maczey E, Riedle S, Garbe C and Schittek B: The adhesion molecule L1 (CD171) promotes melanoma progression. Int J Cancer 119: 549-555, 2006.

18. Silletti S, Yebra M, Perez B, Cirulli V, McMahon M and Montgomery AM: Extracellular signal-regulated kinase (ERK)-dependent gene expression contributes to L1 cell adhesion molecule-dependent motility and invasion. J Biol Chem 279: 28880-28888, 2004.

19. Stoeck A, Gast D, Sanderson MP, Issa Y, Gutwein P and Altevogt P: L1-CAM in a membrane-bound or soluble form augments protection from apoptosis in ovarian carcinoma cells. Gynecol Oncol 104: 461-469, 2007.

20. Sebens Muerkoster S, Lust J, Arlt A, Hasler R, Witt M, Sebens T, Schreiber S, Folsch UR and Schafer H: Acquired chemoresistance in pancreatic carcinoma cells: induced secretion of IL-1beta and NO lead to inactivation of caspases. Oncogene 25: 3973-3981, 2006.

21. Arlt MJ, Novak-Hofer I, Gast D, Gschwend V, Moldenhauer G, Grunberg J, Honer M, Schubiger PA, Altevogt P and Kruger A: Efficient inhibition of intra-peritoneal tumor growth and dissemination of human ovarian carcinoma cells in nude mice by anti-L1-cell adhesion molecule monoclonal antibody treatment. Cancer Res 66: 936-943, 2006.

22. Knogler K, Grunberg J, Zimmermann K, Cohrs S, Honer M, Ametamey S, Altevogt P, Fogel M, Schubiger PA and Novak-Hofer I: Copper-67 radioimmunotherapy and growth inhibition by anti-L1-cell adhesion molecule monoclonal antibodies in a therapy model of ovarian cancer metastasis. Clin Cancer Res 13: 603-611, 2007.

23. Primiano T, Baig M, Maliyekkel A, Chang BD, Fellars S, Sadhu J, Axenovich SA, Holzmayer TA and Roninson IB: Identification of potential anticancer drug targets through the selection of growth-inhibitory genetic suppressor elements. Cancer Cell 4: 41-53, 2003.

24. Moore PS, Sipos B, Orlandini S, Sorio C, Real FX, Lemoine NR, Gress T, Bassi C, Kloppel G, Kalthoff H, Ungefroren H, Lohr M and Scarpa A: Genetic profile of 22 pancreatic carcinoma cell lines. Analysis of K-ras, p53, p16 and DPC4/Smad4. Virchows Arch 439: 798-802, 2001.

25. Gast D, Riedle S, Kiefel H, Sebens Muerkoster S, Schafer H, Schafer MKE and Altevogt P: The RGD integrin binding site in human L1-CAM is important for nuclear signaling. Exp Cell Res 314: 1053-1062, 2008.

26. Arlt A, Vorndamm J, Breitenbroich M, Folsch UR, Kalthoff H, Schmidt WE and Schafer H: Inhibition of NF-kappaB sensitizes human pancreatic carcinoma cells to apoptosis induced by etoposide (VP16) or doxorubicin. Oncogene 20: 859-868, 2001.

27. Schafer H, Arlt A, Trauzold A, Hunermann-Jansen A and Schmidt WE: The putative apoptosis inhibitor IEX-1L is a mutant nonspliced variant of p22(PRG1/IEX-1) and is not expressed in vivo. Biochem Biophys Res Commun 262: 139-145, 1999.

28. Wey JS, Gray MJ, Fan F, Belcheva A, McCarty MF, Stoeltzing O, Somcio R, Liu W, Evans DB, Klagsbrun M, Gallick GE and Ellis LM: Overexpression of neuropilin-1 promotes constitutive MAPK signalling and chemoresistance in pancreatic cancer cells. Br J Cancer 93: 233-241, 2005.

29. Duczmal A, Schollhammer S, Katich S, Ebeling O, SchwartzAlbiez R and Altevogt P: The L1 adhesion molecule supports alpha $\mathrm{v}$ beta 3-mediated migration of human tumor cells and activated T lymphocytes. Biochem Biophys Res Commun 232: 236-239, 1997.

30. Friedl P, Hegerfeldt $\mathrm{Y}$ and Tusch M: Collective cell migration in morphogenesis and cancer. Int J Dev Biol 48: 441-449, 2004.

31. Hynes RO: Integrins: versatility, modulation, and signaling in cell adhesion. Cell 69: 11-25, 1992.

32. Estrugo D, Fischer A, Hess F, Scherthan H, Belka C and Cordes N: Ligand bound beta1 integrins inhibit procaspase- 8 for mediating cell adhesion-mediated drug and radiation resistance in human leukemia cells. PLoS ONE 2: e269, 2007.

33. Miyamoto H, Murakami T, Tsuchida K, Sugino H, Miyake H and Tashiro S: Tumor-stroma interaction of human pancreatic cancer: acquired resistance to anticancer drugs and proliferation regulation is dependent on extracellular matrix proteins. Pancreas 28: 38-44, 2004. 
34. Weekes CD, Kuszynski CA and Sharp JG: VLA-4 mediated adhesion to bone marrow stromal cells confers chemoresistance to adherent lymphoma cells. Leuk Lymphoma 40: 631-645, 2001.

35. Weinel RJ, Rosendahl A, Neumann K, Chaloupka B, Erb D, Rothmund $\mathrm{M}$ and Santoso S: Expression and function of VLA-alpha 2, -alpha 3, -alpha 5 and -alpha 6-integrin receptors in pancreatic carcinoma. Int J Cancer 52: 827-833, 1992.

36. Grzesiak JJ and Bouvet M: The alpha2beta1 integrin mediates the malignant phenotype on type I collagen in pancreatic cancer cell lines. Br J Cancer 94: 1311-1319, 2006.

37. Vogelmann R, Kreuser ED, Adler G and Lutz MP: Integrin alpha6beta1 role in metastatic behavior of human pancreatic carcinoma cells. Int J Cancer 80: 791-795, 1999.
38. Sawai H, Funahashi H, Matsuo Y, Yamamoto M, Okada Y, Hayakawa $\mathrm{T}$ and Manabe T: Expression and prognostic roles of integrins and interleukin-1 receptor type I in patients with ductal adenocarcinoma of the pancreas. Dig Dis Sci 48: 1241-1250, 2003.

39. Sawai H, Okada Y, Funahashi H, Matsuo Y, Takahashi H, Takeyama $\mathrm{H}$ and Manabe T: Interleukin-1alpha enhances the aggressive behavior of pancreatic cancer cells by regulating the alpha6beta1-integrin and urokinase plasminogen activator receptor expression. BMC Cell Biol 7: 8, 2006. 